\title{
O uso de serviços de atenção primária à saúde pela população infantil em um estado do nordeste brasileiro
}

\author{
The use of primary attention services to health \\ by the childhood population in a brazilian northeast
}

\author{
Carlos Leonardo Figueiredo Cunha, Raimundo Antonio da Silva², Mônica Elinor Alves \\ Gama $^{3}$, Geny Rose Cardoso Costa ${ }^{1}$, Andrea Suzana Vieira Costa ${ }^{1}$, Sueli Rosina Tonial ${ }^{4}$
}

\begin{abstract}
Resumo
Este trabalho teve como objetivo avaliar a utilização de alguns serviços de Atenção Básica pela população infantil no estado do Maranhão, comparando-se a evolução dos indicadores nos últimos dez anos. Realizou-se um estudo descritivo, no período de julho de 2007 a janeiro de 2008, com uma amostra representativa do estado do Maranhão de 1.711 crianças, obtida por amostragem aleatória sistemática por conglomerados. Foi utilizada a pesquisa "Saúde, Nutrição e Mortalidade Infantil no Maranhão", desenvolvida em 1996, como referencial comparativo aos dados do presente estudo. Do total de crianças estudadas, $38,4 \%$ realizaram a consulta preventiva no primeiro ano de vida, das quais $92,1 \%$ utilizaram o Sistema Único de Saúde (SUS). Observou-se que praticamente todas as variáveis tiveram incremento positivo em relação ao estudo da década passada - as consultas de puericultura no primeiro ano de vida e antes do primeiro mês de vida em média $10 \%$; já o acesso ao serviço público ascendeu em torno de 17\%. Conclusões: Com essa análise do comportamento atual e de 10 anos atrás, observou-se a melhoria em alguns indicadores de atenção à saúde da criança no estado do Maranhão, possivelmente relacionada aos processos de implantação e aprimoramento da Estratégia Saúde da Família, determinantes do processo de consolidação do Sistema Público de Saúde no Brasil - evolução que pode parecer lenta aos olhos das pesquisas, mas que tem a dimensão necessária para configurar-se na maior política de inclusão social do país.
\end{abstract}

Palavras-chave: saúde da criança; atenção primária à saúde; sistema único de saúde.

\begin{abstract}
This paper has as its general goal to avaliate the use of some Basic Attention's service by the childhood population in the State of Maranhão (Brazil), when compand to the evolution of the indicators in the last ten years. It was performed a descriptive study in the period from july of 2007 to January of 2008 and used a sample which represented the state of Maranhão among 1.711 children. It was obtained according to systematical aleatory sampling for conglomerates. It was used the Research "Health, Nutrition and Infantile Mortality in Maranhão" developed in 1996, like data comparative referential the present study. From the total of studied children 38\% got their preventive consultation in their 1st year of life from which $92,1 \%$ used the Health Unique System (SUS). It was observed that all the variables had their positive increase as to relation to the study of the past decade, practically. The pericultural consultations in the 1st year of life and before the 1st month of life in an average of $10 \%$ however the access to the public service used to $17 \%$ approximately. Throng that analysis of the behavior and that
\end{abstract}

Trabalho realizado na Universidade Federal do Maranhão (UFMA), São Luís (MA), Brasil.

'Mestre em Saúde Materno-Infantil pela UFMA - São Luís (MA), Brasil.

²Doutor em Saúde Pública pela Universidade de São Paulo (USP) - São Paulo (SP), Brasil; Professor Associado do Departamento de Saúde Pública da UFMA -

São Luís (MA), Brasil.

${ }^{3}$ Doutora em Medicina pela USP - São Paulo (SP), Brasil; Professora Adjunta da Universidade Federal do Maranhão UFMA - São Luís (MA), Brasil. ${ }^{4}$ Doutora em Saúde Materno-Infantil pela FIOCRUZ - Rio de Janeiro (RJ); Professora Adjunta da Universidade Federal do Maranhão (UFMA) - São Luís (MA), Brasil. Endereço para correspondência: Carlos Leonardo Figueiredo Cunha - Universidade Federal do Maranhão - Rua do Aririzal, quadra 01, casa 09 - CEP: $65073-670$ São Luís (MA), Brasil - E-mail: leocunhama@gmail.com

Fonte de financiamento: Fundação de Amparo à Pesquisa e Desenvolvimento Científico do Maranhão (FAPEMA), Conselho Nacional de Desenvolvimento

Científico e Tecnológico (CNPq).

Conflito de interesse: nada a declarar. 
one of 10 years before, it was observed the improvement of some indicators of the child's health attention in the state of Maranhão, possibly related to the processes of implantation and betterment of the Family's Health Strategy, so determinants of the process of consolidation of Brazil's Public Health System - evolution which can seem slow to the eyes of the researches, however with a necessary dimention in order to configurate itself in the major politics of social inclusion of the Country.

Keywords: child health; primary health careba; unified health system.

\section{INTRODUÇÃO}

Sabe-se que um dos grandes contingentes para o sistema público de saúde é a população infantil, que demanda cuidados específicos para a garantia de sua saúde. Os menores de um ano e a população idosa apresentam os maiores riscos de internação hospitalar por condições sensíveis à atenção primária $(\mathrm{CSAP})^{1}$. Atualmente, tem-se utilizado as taxas de internações por CSAP como indicador de qualidade de acesso ou desempenho dos serviços de atenção primaria, reconhecendo-se que o excesso de hospitalizações representa um sinal de alerta e deve acionar mecanismos de análise e busca de estratégias para melhoria das ações nesse nível de atenção ${ }^{2}$.

Apesar da institucionalização do Programa Saúde da Família, desde 1994, como principal estratégia de organização da Atenção Básica, o seu impacto nos indicadores de saúde permanece controverso. Nota-se uma relação direta desses indicadores com a situação socioeconômica, confirmando a importância da determinação social nos agravos que acometem a população infantil ${ }^{3,4}$.

Porém, existe uma clara relação entre a qualidade dos serviços de atenção primária à saúde da criança e os diferentes contextos locorregionais ${ }^{5,6}$. No Maranhão, a Estratégia Saúde da Família possui uma cobertura de $77,1 \%$ e, no entanto, ainda possui alta taxa de mortalidade infantil $(21,9 \%$ ) em relação à média brasileira $(16 \%))^{7}$. Os percentuais de crianças maranhenses com esquema vacinal básico, com aleitamento materno exclusivo e de cobertura de consultas de pré-natal, são inferiores tanto à média nacional como à da região Nordeste. Assim, os agravos relacionados a esses fatores de proteção, ocorrem com maior frequência8.

Em um contexto de desigualdades sociais e escassez de recursos públicos para o financiamento do setor saúde, os impactos provocados pelas políticas de saúde nem sempre resultam em mudanças esperadas ou atendem às necessidades e expectativas da população. Nesse sentido, a avaliação é de suma importância para mensurar a capacidade de respostas de políticas, programas e serviços às necessidades de saúde da população?.

Vale ressaltar que a produção científica nacional carece de referências atuais acerca da utilização de serviços de saúde pela população infantil, em especial, no que se refere aos serviços de puericultura.
No estado do Maranhão, são poucas as informações existentes sobre a situação de saúde atual da população infantil e do uso dos serviços de saúde. Os dados coletados na rotina das instituições de saúde são insuficientes para se ter uma visão mais completa sobre o panorama sanitário, pois, em geral, caracterizam-se pelo sub-registro e inconsistências dos dados.

No âmbito estadual, o trabalho mais completo sobre a saúde da criança foi a Pesquisa Estadual de Saúde e Nutrição e a Pesquisa Saúde, nutrição e mortalidade infantil no Maranhão realizadas em 1991 e 1997 respectivamente, com o objetivo de realizar um diagnóstico da situação de saúde e nutrição das crianças menores de cinco anos ${ }^{10,11}$. O presente artigo teve como objetivo avaliar a utilização dos serviços de Atenção Básica pela população infantil no estado do Maranhão, comparando-se a situação atual com dados de pesquisa realizada há 10 anos, nos mesmos moldes em nosso estado.

\section{MÉTODOS}

Realizou-se estudo transversal, com abordagem quantitativa, procedendo-se a coleta de dados nos meses de julho de 2007 a janeiro de 2008. Esse estudo trata-se de um recorte de uma grande pesquisa de base populacional no estado do Maranhão com o objetivo maior de avaliar a Situação de Saúde Materno-Infantil no estado. O foco do presente recorte é a avaliação da utilização dos serviços de saúde pela população infantil; a amostra é representativa do estado do Maranhão, perfazendo o total de 1.711 crianças de zero a cinco anos.

O evento considerado para definição do tamanho amostral foi "Desnutrição" (24\% de ocorrência), tendo-se como referencial o resultado encontrado para essa variável em pesquisa também de base populacional realizada por Tonial e Silva ${ }^{11}$, no Maranhão. A amostra calculada foi de 1.711 crianças, obtida por amostragem aleatória sistemática por conglomerados; considerando o tamanho amostral, foram entrevistadas 57 crianças em cada conglomerado. Com este tamanho de amostra, o estudo foi capaz de avaliar os indicadores de saúde com uma margem de erro, em geral, inferior a $3 \%$. O efeito do desenho foi de $2 \%$, o intervalo de confiança de $95 \%$, nível de significância de $5 \%$ e precisão das estimativas de $3 \%$. Não houve cálculo amostral diferenciando faixas etárias.

Utilizou-se um processo de amostragem aleatória sistemática de conglomerados, em estágios múltiplos, com três etapas. 
Na primeira etapa foram sorteados os municípios do estado do Maranhão, sendo elaborada uma listagem acumulada de municípios e suas respectivas populações, estimadas para o ano de 2006, de acordo com o último Censo Demográfico do Instituto Brasileiro de Geografia e Estatística (IBGE), disponibilizado pela instituição.

Foram sorteados 30 conglomerados com vistas a uma distribuição normal. O processo de amostragem por conglomerados possibilitou que os municípios com maior população tivessem maior probabilidade de serem escolhidos, ou até sorteados mais de uma vez, como ocorreu em São Luís, capital do estado, que possui três conglomerados computados no estudo. Os 28 municípios sorteados apresentam-se distribuídos aleatoriamente nas regiões geográficas do estado.

No segundo estágio foram sorteados os setores censitários dentro de cada município, a partir de uma listagem dos setores fornecida pelo IBGE. Em cada conglomerado foram sorteados sete setores para visitação e mais dois setores reservas, a serem utilizados em casos excepcionais, quando da impossibilidade de acesso ao setor sorteado, pois a pesquisa estava prevista para ser realizada em período chuvoso, passível de problemas de acesso geográfico. No terceiro estágio foi sorteado o ponto inicial dentro de cada setor, a partir do qual certo número de domicílios foi visitado. No caso de recusa, óbito ou domicílio sem criança na faixa etária do estudo, domicílios conseguintes foram visitados até completar a amostra.

$\mathrm{O}$ instrumento de coleta de dados foi um questionário contendo variáveis específicas da criança construídas no projeto "Situação de Saúde no Estado do Maranhão", sendo as definições utilizadas presentes no manual do entrevistador. Foi desenvolvido um projeto-piloto para garantir unidade no momento da coleta das informações e desenvolvimento das etapas definidas para o trabalho de campo. Foram selecionadas, para essa análise, variáveis referentes à utilização dos serviços de saúde no âmbito da atenção primaria à saúde da criança (consulta de puericultura: no primeiro ano de vida, antes do primeiro mês de vida, formas de financiamento; posse do cartão/caderneta da criança, registro da pesagem e profissional que realizou a pesagem). Priorizou-se a mãe como informante ou o responsável direto pelos cuidados com a criança. Para todos os dados coletados, foram consideradas as informações prestadas convalidadas quando descritas no cartão/caderneta da criança, visando reduzir viés de memória dado o período transcorrido.

Foram utilizados percentuais descritivos. Para digitação e processamento de dados foi utilizado o programa Epi-Info 2.002 do CDC, de Atlanta, EUA ${ }^{12}$. Para avaliar as mudanças transcorridas nos últimos dez anos, foram considerados os resultados observados na pesquisa "Saúde, nutrição e mortalidade infantil no Maranhão", realizada em 1996, cuja estruturação metodológica e desenvolvimento seguiram o mesmo padrão da pesquisa atual.

A presente pesquisa foi submetida e aprovada pelo Comitê de Ética em Pesquisa do Hospital Universitário da Universidade Federal do Maranhão sob o registro no $33104-747 / 2006$, sendo emitido o parecer favorável para realização da pesquisa em 20 de outubro de 2006.

\section{RESULTADOS}

O perfil epidemiológico das 1.711 crianças revelou baixa escolaridade materna ( $24 \%$ com até quatro anos de estudo), renda familiar baixa (58,8\% com até um salário mínimo). Em relação a escolaridade do pai predominou a de um a quatro anos $(24,9 \%)$ (Tabela 1$)$.

$\mathrm{Na}$ Tabela 2, estão dispostas as características em relação à consulta de puericultura: sua realização no primeiro ano de vida ocorreu entre $38,4 \%$ das crianças, sofrendo incremento positivo de 11,4\% de 1996 a 2007; a consulta antes do primeiro mês de vida ficou em 35,6\%, alcançando um incremento um pouco menor (10,9\%) em relação ao dado de 1996.

Aos que fizeram consultas de puericultura, foi questionada a forma de financiamento observando-se que a maioria acessou o Sistema Único de Saúde (SUS), com aumento de $13,5 \%$ em relação à última década. Do total de crianças que fez consulta no sistema público de saúde, $67,8 \%$ o fizeram em Unidade Básica de Saúde da Família (Tabela 2).

Analisando a utilização de serviços de puericultura pela população em estudo (Tabela 3), observa-se que a maioria $(61,4 \%)$ não realizou consulta médica ou de enfermagem. Dentre as que relataram a realização, a maioria destas consultas foi feita na faixa etária de um a seis meses $(55,8 \%)$, nos serviços do Sistema Único de Saúde $(92,1 \%)$, com predominância na Unidade Básica de Saúde da Família (UBSF) $(67,8 \%)$.

A procura por serviços de saúde assistenciais (decorrentes de queixas agudas relatadas nos últimos três meses) foi de $53,3 \%$, sendo que, destas, $99,3 \%$ conseguiram realizar a consulta ainda nesse período de três meses. Mais de 90\% dessas consultas foram no SUS e, destas, 67,8\% em Unidade Básica de Saúde da Família. Na pesquisa de 10 anos atrás, menos de $40 \%$ relatavam realização de consulta no mesmo período e pouco mais de $70 \%$ em serviços públicos (Tabela 4 ).

\section{DISCUSSÃO}

A atenção à saúde da criança no primeiro ano de vida é essencial para o seu crescimento e desenvolvimento saudável; 
para garantir, no âmbito do SUS, ações focadas nessa faixa etária, em 2004, o Ministério da Saúde lançou a "Agenda de Compromissos para a Saúde Integral da Criança e Redução da Mortalidade Infantil"13 o que vem influenciando positivamente a organização dos serviços voltados a população infantil.

Tabela 1. Distribuição das 891 mães de crianças entrevistadas, segundo as características socioeconômicas. Maranhão, 2007

\begin{tabular}{|c|c|c|}
\hline Variáveis & $\mathbf{n}$ & $\%$ \\
\hline \multicolumn{3}{|c|}{ Situação conjugal da mãe/cuidador } \\
\hline Solteira & 213 & 23,9 \\
\hline União consensual & 494 & 55,4 \\
\hline Casada & 127 & 14,3 \\
\hline Divorciada & 35 & 3,9 \\
\hline Viúva & 17 & 1,9 \\
\hline Outro & 5 & 0,6 \\
\hline Total & 891 & 100,0 \\
\hline \multicolumn{3}{|l|}{ Ocupação da Mãe/Cuidador } \\
\hline Do Lar & 493 & 55,3 \\
\hline Empregada Doméstica & 100 & 11,2 \\
\hline Comerciaria & 34 & 3,8 \\
\hline Serviços gerais & 32 & 3,6 \\
\hline Em casa, para fora & 22 & 2,5 \\
\hline Outro & 210 & 23,5 \\
\hline Total & 891 & 100,0 \\
\hline \multicolumn{3}{|l|}{ Escolaridade mãe } \\
\hline 5 a 8 anos & 297 & 33,3 \\
\hline 9 a 13 anos & 251 & 28,2 \\
\hline 1 a 4 anos & 214 & 24,0 \\
\hline Sem escolaridade & 101 & 11,3 \\
\hline Superior completo & 10 & 1,1 \\
\hline Superior incompleto & 9 & 1,0 \\
\hline Ignorado & 9 & 1,0 \\
\hline Total & 891 & 100,0 \\
\hline \multicolumn{3}{|l|}{ Escolaridade do Cônjuge } \\
\hline 1 a 4 anos & 222 & 24,9 \\
\hline 5 a 8 anos & 204 & 22,9 \\
\hline 9 a 13 & 184 & 20,7 \\
\hline Sem escolaridade & 178 & 20,0 \\
\hline Ignorado & 84 & 9,4 \\
\hline Superior incompleto & 10 & 1,1 \\
\hline Superior completo & 9 & 1,0 \\
\hline Total & 891 & 100,0 \\
\hline \multicolumn{3}{|l|}{ Renda familiar } \\
\hline Até 1 salário* & 523 & 58,8 \\
\hline 1 a 1.9 salários & 192 & 21,6 \\
\hline 2 a 4,9 salários & 140 & 15,7 \\
\hline 5 a 9,9 salários & 17 & 1,9 \\
\hline Ignorado & 10 & 1,2 \\
\hline Não se aplica & 5 & 0,6 \\
\hline 10 e mais salários & 4 & 0,2 \\
\hline Total & 891 & 100,0 \\
\hline
\end{tabular}

*Salário mínimo de 2007 - R\$ 380,00
Porém, a realidade socioeconômica encontrada no Maranhão deve ser considerada como fator determinante de sua situação de saúde e de acesso aos serviços preventivos ou assistenciais, o que tem colocado o estado em uma posição desfavorável em relação aos indicadores de saúde ${ }^{14}$.

A renda familiar e baixa escolaridade inegavelmente interferem no acesso a serviços de saúde ${ }^{15}$. A grande quantidade de filhos e o curto espaço interpartal também podem influenciar no acompanhamento de saúde durante o primeiro ano de vida dessas crianças, pois em famílias com muitas crianças, as mães tendem a considerar como prioridade apenas o adoecimento ${ }^{16}$.

Contrapondo que a determinação socioeconômica é um fator determinante ao acesso aos serviços de saúde, um estudo realizado em usuários cobertos pela Estratégia Saúde da Família em Porto Alegre evidenciou uma maior utilização da Unidade Básica de Saúde entre pessoas com menor nível socioeconômico e sem cobertura por plano de saúde ${ }^{17}$.

Nesse estudo, destaca-se o incremento positivo no percentual de crianças que compareceram a consultas de puericultura no primeiro ano de vida em relação à década passada. Porém, observando-se estudos nacionais constata-se que o percentual de crianças com consulta de puericultura em nosso estado ainda é baixo. Estudo realizado sobre a evolução da assistência materno-infantil na cidade de São Paulo revelou que as consultas de prevenção no primeiro ano de vida em 1984 foram equivalentes a 76\% e em 1996 atingiu 84,2\% da população ${ }^{18}$. Em Niterói/RJ, 82\% das mães referem levar, por rotina, seus filhos para monitoramento da saúde no primeiro ano de vida ${ }^{19}$. Em Pelotas/RS, o percentual de crianças que realizaram consultas preventivas no primeiro ano de vida, em 1996 , foi de $98,5 \%{ }^{20}$.

Diversos fatores estão relacionados à cobertura da puericultura, frequentemente associados ao acesso aos serviços de saúde, como as condições geográficas (distância, manutenção das

Tabela 2. Evolução dos indicadores de utilização de serviços preventivos pela população infantil na última década - Pesquisas "Saúde, nutrição e mortalidade infantil no Maranhão" - 1996 e "Situação de Saúde no Maranhão" - 2007

$\begin{array}{lcc}\text { Variáveis } & 1996(\%) & 2007(\%) \\ \begin{array}{l}\text { Consulta de puericultura no primeiro } \\ \text { ano de vida }\end{array} & 27,0 & 38,4 \\ \begin{array}{l}\text { Consulta de puericultura antes do } \\ \text { primeiro mês de vida }\end{array} & 24,7 & 35,6 \\ \begin{array}{l}\text { Consultas no SUS } \\ \text { Consultas em Unidade de Saúde da }\end{array} & 78,6 & 92,1 \\ \begin{array}{l}\text { Família } \\ \text { Consultas em serviços privados }\end{array} & - & 67,8 \\ \begin{array}{l}\text { Consultas em serviços privados e/ou } \\ \text { filantrópicos conveniados ao SUS }\end{array} & 12,9 & 2,0 \\ \end{array}$


Tabela 3. Distribuição das 891 mães de crianças entrevistadas, segundo a utilização de serviços de puericultura pela população infantil. Maranhão, 2007

\section{Variáveis}

n $\%$

Realização de consulta de puericultura

$\begin{array}{lcc}\text { Não fez } & 547 & 61,4 \\ \text { Sim } & 342 & 38,4 \\ \text { Não sabe } & 2 & 0,2 \\ \text { Total } & 891 & 100,0\end{array}$

Idade da criança na $1^{\text {a }}$ consulta de puericultura

$\begin{array}{lcc}1-6 \text { meses } & 191 & 55,8 \\ <1 \text { mês } & 122 & 35,6 \\ 7-12 \text { meses } & 16 & 4,6 \\ \text { Ignorado } & 12 & 3,8 \\ >12 \text { meses } & 1 & 0,2 \\ \text { Total } & 342 & 100,0\end{array}$

Financiamento da $1^{\text {a }}$ consulta de puericultura

$\begin{array}{lcc}\text { SUS } & 315 & 92,1 \\ \text { Particular } & 12 & 3,5 \\ \text { Usou convênio ou seguro-saúde } & 7 & 2,0 \\ \text { SUS conveniado ou filantrópico } & 6 & 1,8 \\ \text { Ignorado } & 2 & 0,6 \\ \text { Total } & 342 & 100,0\end{array}$

Realização da $1^{\text {a }}$ consulta de puericultura em UBSF

\begin{tabular}{lcc} 
Sim & 232 & 67,8 \\
Não & 98 & 28,6 \\
Ignorado & 12 & 3,6 \\
Total & 342 & 100,0 \\
\hline
\end{tabular}

Tabela 4. Evolução dos indicadores de utilização de serviços assistenciais pela população infantil na última década - Pesquisas "Saúde, nutrição e mortalidade infantil no Maranhão" - 1996 e "Situação de Saúde no Maranhão" - 2007

\begin{tabular}{lcc} 
Variáveis & $1996(\%)$ & $2007(\%)$ \\
$\begin{array}{l}\text { Procura por consulta médica nos últimos } \\
\text { três meses }\end{array}$ & - & 53,3 \\
$\begin{array}{l}\text { Consulta médica realizada nos últimos } \\
\text { três meses }\end{array}$ & 35,2 & 99,3 \\
$\begin{array}{l}\text { Consulta no SUS } \\
\text { Consulta em Unidade de Saúde da }\end{array}$ & 74,7 & 94,9 \\
$\begin{array}{l}\text { Família } \\
\text { Consulta em serviços privados }\end{array}$ & - & 67,8 \\
$\begin{array}{l}\text { Consulta em serviços privados e/ou } \\
\text { filantrópicos conveniados ao SUS }\end{array}$ & 13,0 & 2,2 \\
\hline
\end{tabular}

estradas e frota de transporte coletivo) e o meio de transporte disponível ${ }^{21,22}$. A não realização de consultas de puericultura no Maranhão pode estar associada às diferentes características das regiões do estado, que tem grandes dimensões geográficas e, em geral, possuem municípios de pequeno porte, distantes de grandes centros urbanos e com baixa densidade demográfica. $\mathrm{Na}$ capital do estado, a população, já de longa data, apresenta outro perfil, como mostra uma pesquisa realizada em São Luís em 1994, que revelou que $80 \%$ das crianças haviam comparecido a consultas de puericultura no primeiro ano de vida ${ }^{23}$.

No entanto, estudo realizado em municípios nordestinos de grande porte não identificou diferenças significativas nos indicadores de saúde da criança entre áreas cobertas e não cobertas pelo PSF. O impacto do Programa Saúde da Família nos indicadores de saúde nos municípios pode variar de acordo como o seu contexto de implantação e condução e das características municipais no campo socioeconômico e de políticas públicas, evidenciando a influência da determinação social nos agravos à saúde $e^{3,4}$.

Ao avaliarmos o uso dos serviços públicos observamos o aumento percentual que se justifica pela progressiva consolidação do sistema público de saúde no Brasil, inclusive com a implantação da Estratégia Saúde da Família e sua progressiva ampliação de cobertura em áreas de maior risco social ${ }^{13,24}$. Atualmente, gira em torno de $77,1 \%{ }^{25}$ a cobertura da Estratégia Saúde da Família no Maranhão, o que reflete a utilização desses serviços para consulta de puericultura em $67,8 \%$ na presente pesquisa.

Analisando-se evolutivamente os indicadores de atenção à saúde da criança, pode-se sugerir que os incrementos positivos podem ser relacionados à abrangência das politicas sociais em nosso país e ao fortalecimento da Estratégia Saúde da Família na última década que, no Maranhão, tem apresentado um padrão de expansão acelerada de cobertura — em 1994 (0,5\%), em 1996 (2\%) e em $2007(75,5 \%)^{26}$. O fortalecimento do SUS ao longo da última década se confirma em nossa pesquisa onde mais de $90 \%$ da população utiliza os serviços públicos de saúde, corroborando estudos que mostram que o SUS atende a mais de 150 milhões de brasileiros - evolução que pode parecer lenta aos olhos das pesquisas, mas que tem a dimensão necessária para configurarse na maior política de inclusão social do país ${ }^{27,28}$.

Deve-se destacar, como limite na interpretação dos dados, a representatividade da amostra, considerando-se que indicadores do estado nutricional demonstram taxas de desnutrição para o estado do Maranhão, atualmente, inferiores a selecionada para cálculo à época do estudo, bem como a não inclusão de dados de crianças que evoluíram a óbito. 


\section{REFERÊNCIAS}

1. Perpétuo IHO, Wong LR. Atenção hospitalar por condições sensíveis à atenção ambulatorial (CSAA) e as mudanças no seu padrão etário: uma análise exploratória dos dados de Minas Gerais. In: Procedimento do $12^{\circ}$ Seminário sobre a Economia Mineira; 2006. Minas Gerais; 2006.

2. Alfradique ME, Bonolo PF, Dourado I, Lima-Costa MF, Macinko J, Mendonça CS, et al. Internações por condições sensíveis à atenção primária: a construção da lista brasileira como ferramenta para medir o desempenho do sistema de saúde (Projeto ICSAP - Brasil). Cad Saúde Pública. 2009; 25(6):1337-49.

3. Conil EM. Ensaio histórico-conceitual sobre a Atenção Primária à Saúde: desafi os para a organização de serviços básicos e da Estratégia Saúde da Família em centros urbanos no Brasil. Cad Saúde Pública. 2008;24(Suppl 1):S7-S27.

4. Roncalli AG, Lima KC. Impacto do Programa Saúde da Família sobre indicadores de saúde da criança em município de grande porte da região Nordeste do Brasil. Ciênc Saúde Coletiva. 2006;11:713-24.

5. Campos CEA. Estratégias de avaliação e melhoria contínua da qualidade no contexto da Atenção Primária à Saúde. Rev Bras Saúde Matern Infant. 2005;5(Suppl 1): S63-S69.

6. Sarti TD, Lima RCD, Silva MZ, Maciel ELN. A institucionalização da avaliação na atenção básica: alguns aspectos para a construção de práticas democráticas no contexto da estratégia saúde da família. UFES Rev Odont. 2008;10(1):63-6.

7. Instituto Brasileiro de Geografia e Estatística (IBGE). Síntese de indicadores sociais 2010: uma análise das condições de vida da população Brasileira. Rio de Janeiro: IBGE; 2011 [internet]. Disponível em: http:// www.ibge.gov.br/estadosat/temas.php?sigla=pa\&tema=sis_2010

8. Brasil. Ministério da Saúde. Secretaria Executiva. Banco de Dados Sistema Único de Saúde. Cadernos de Informações de Saúde Informações de Saúde - Informações Gerais. Brasília; 2011 [internet]. Disponível em: http://tabnet.datasus.gov.br/tabdata/cadernos/brasil.htm

9. Facchini LA, Tomasi E, Thumé E, Teixeira VA, Silveira DS, Maia MFS, et al. Avaliação de efetividade da Atenção Básica à Saúde em municípios das regiões Sul e Nordeste do Brasil: contribuições metodológicas. Cad Saúde Pública. 2008;24(Suppl 1):S159-S172.

10. Brasil. Fundo das Nações Unidas para a Infância. Saúde e nutrição das crianças nordestinas: pesquisas estaduais 1987-1992. Brasília: FUNICEF; 1995.

11. Tonial SR, Silva AAM. Saúde, nutrição e mortalidade infantil no Maranhão. São Luís: UFMA; 1997.

12. Dean AG, Dean JA, Burton AH, Dicker RC. Epinfo, versão 6: um sistema de processamento de texto, banco de dados e estatística para computadores. São Paulo: Centers of Disease Control; 1994.

13. Brasil. Ministério da Saúde. Termo de referência para o estudo de linha de base dos municípios selecionados para o componente I do PROESF. Brasília: MS; 2004.
14. Sousa TRV, Leite Filho PAM. Análise por dados em painel do status de saúde no Nordeste Brasileiro. Rev Saúde Pública. 2008;42(5):796-804.

15. Coimbra LC, Silva AAM, Mochel EG, Alves MTSSB, Ribeiro VS, Aragão VMF, Bettiol H. Fatores associados à inadequação do uso da assistência pré-natal. Rev Saúde Pública. 2003;37(4):456-62.

16. Costa MCN, Mota ELA, Paim JS, Silva LMV, Teixeira MG, Mendes CMC. Mortalidade infantil no Brasil em períodos recentes de crise econômica. Rev. Saúde Pública. 2003;37(6):699-706.

17. Fernandes LCL, Bertoldi AD, Barros AJD. Utilização dos serviços de saúde pela população coberta pela Estratégia de Saúde da Família. Rev Saúde Pública. 2009;43:595-603.

18. Monteiro CA, França Junior I, Conde WL. Evolução da assistência materno-infantil na cidade de São Paulo (1984-1996). Rev Saúde Pública. 2000;34(6):19-25.

19. Barroso SE, Miasato JM, Graça TCA. Avaliação de freqüência de visitas ao pediatra $\mathrm{x}$ visitas ao odontopediatra em unidade básica de saúde do município de Niterói-RJ. J Bras Odontoped Odonto Bebe. 2001;3(14):324-7.

20. Costa JSD, Madeira ACC, Manzolli PP, Luz RM, Britto MAP, Sallaberry DD. Diagnóstico das condições de saúde e assistência médica das crianças na Vila Santos Dumont, Pelotas, RS. Revista AMRIGS. 2001;4(1,2):33-9.

21. Blank D. A puericultura hoje: um enfoque apoiado em evidências. J Pediatr (Rio J). 2003;79(Suppl 1):S13-S22.

22. Holanda F. Dinâmica da economia maranhense nos últimos 25 anos. São Luís: ISMEC; 2008.

23. Silva AAM, Gomes UA, Tonial SR, Silva RA. Cobertura de puericultura e fatores associados em São Luís (Maranhão), Brasil. Rev Panam Salud Publica. 1999;6(4):266-72.

24. Brasil. Conselho Nacional de Secretários de Saúde. Sistema Único de Saúde. Brasília: CONASS; 2007. (Coleção Progestores - Para entender a gestão do SUS, 12).

25. Brasil. Ministério da Saúde. Departamento de Atenção Básica. Teto, credenciamento e implantação das estratégias de Agentes Comunitários de Saúde, Saúde da Família e Saúde Bucal: Maranhão: 2012. [internet]. Disponível em: http:// dab.saude.gov.br/historico_cobertura_sf/historico_cobertura_sf_relatorio.php

26. Brasil. Ministério da Saúde. Cobertura do Programa Saúde da Família: Maranhão. Brasília; 2009. [internet]. Disponível em: http://www.saude. gov.br/dab.

27. Brasil. Conselho Nacional de Secretários de Saúde. SUS: avanços e desafios. Brasília, DF: CONASS, 2006

28. Santos NR. Desenvolvimento do SUS, rumos estratégicos e estratégias para visualização dos rumos. Ciênc Saúde Coletiva. 2007;12(2):429-35.

Recebido em: 24/06/2012 Aprovado em: 29/05/2013 